\title{
Analysis of impedance measurements of a suspension of microcapsules using a variable length impedance measurement cell
}

\author{
Dejan Križaj ${ }^{1,2}$, Borut Pečar ${ }^{1}$ \\ Faculty of Electrical Engineering, University of Ljubljana, Ljubljana, Slovenia \\ E-mail any correspondence to: dejan.krizaj@fe.uni-lj.si
}

\begin{abstract}
Electrical impedance measurements of a suspension have to take into account the double layer impedance that results from a very thin charged layer formed at the electrode-electrolyte interface. A dedicated measuring cell that enables variation of the distance between the electrodes was developed to investigate the electrical properties of suspensions using two-electrode impedance measurements. By varying the distance between the electrodes it is possible to separate the double layer and the suspension impedance from the measured data. Electrical 'lumped' models have been developed from measured and extracted impedances. The error of non-inclusion of the double layer impedance has been analyzed. The error depends on the frequency of the measurement as well as the distance between the electrodes.
\end{abstract}

Keywords: Suspension impedance, double layer, paraffin microcapsules, lumped model.

\section{Introduction}

Impedance spectroscopy measurements of electrolytes are frequently difficult to perform due to a thin charged layer (double layer) that forms between the electrode and the media [1, 2]. The double layer is a result of charge redistribution close to the electrode that results in a very thin layer of high capacitance disturbing the impedance measurements of the media (particularly at low frequencies). We have investigated the possibility of eliminating the double layer effect by constructing a measurement cell capable of varying the distance between the electrodes. This possibility of elimination of the electrode polarization effect using two-electrode impedance measurements was described by Fricke and Curtis in the 1930s [3] and later used frequently by Geddes and others [4]. Similar cells are also used for measuring permittivity and permeability of lossy materials [5]. Several other methods of electrode polarization elimination (reduction) are known, such as a substitutional method using a calibration solution (see for instance [6]), four-electrode measurements [7, 8], increased current density method [9], electrode modifications [10], reduction of sample conductivity etc. Some methods of reduction and correction of electrode properties have been recently reviewed in more detail by Kalvoy et al [11].

A capacitor is clearly a very simplified model for description of the electrical properties of the double layer. In actual practice the double layer should be regarded as a nonideal capacitor (with voltage lagging the current) that can include inductive and resistive elements. The impedance of the electrode/electrolyte has been largely investigated already by Geddes [2] and is covered in several books [12-14]. Mostly, the phenomena is explained and discussed through a dispersion of relaxation times that can be described by the Cole-Cole model or similar equations. In our investigation we modeled the electrode/electrolyte "capacitor" as a series combination of a resistor and a constant phase element. More sophisticated models could possibly improve the fitting error but at the price of added complexity.

The procedure used in this research is cumbersome to a certain extent as it involves mechanically operating the measurement cell in order to vary the distance between the electrodes. Nevertheless, a similar approach can be applied by developing a measurement cell that has a series of planar measuring strips, for instance, with the impedance measured between the two strips of different separation. The impedance of the suspension between the strips will not vary linearly with the distance between the strips, however, though numerical modeling or experiments one can easily determine the cell constants.

The aim of the investigation was to develop and analyze a measurement cell that enabled (bio)impedance measurements by varying the distance between the electrodes. One possible use of the developed measurement cell is in the analysis of the electrical properties of a suspension of paraffin microcapsules. Such a suspension was also used as a sample in our investigation. By assuming that the double layer impedance remained unaffected during changes in the distance between the electrodes and that the impedance of the media between the electrodes changed linearly with the distance between the electrodes, it was possible to separate the measurement results from those of the double layer impedance and the media. From extracted data, a lumped electrical model of a suspension with microcapsules was developed. In the final part of this paper we present an analysis of the errors involved in determining the relevant parameters when the double layer impedance was not taken into account.

\section{Materials and methods}

A dedicated measurement cell was developed using technology for acrylic glass manipulation. The 
measurement setup with a measurement cell is shown in Figure 1. The measurement cell consists of two electrodes placed in parallel of area $4 \mathrm{~cm}^{2}$ each. The screw enables variation of the distance between the electrodes from 0 to $10 \mathrm{~cm}$ with an accuracy of approximately $0.5 \mathrm{~mm}$. The reason for vertical placement of the electrodes was that with this approach we minimized the problems associated with liquid leakage and sealing.

A suspension of microcapsules was used as a medium. Microencapsulations are frequently used in pharmaceutical industries for encapsulation of drugs. The paraffin microcapsules are of interest in particular for their ability to store thermal energy. Their usage is thus seen in the thermal insulation industry or for storage of the solar energy [15]. The paraffin microcapsules studied (see Figure 2) were suspended in $0.1 \mathrm{M} \mathrm{KCl}$. The microcapsules varied in diameter from a few microns up to tens of microns.

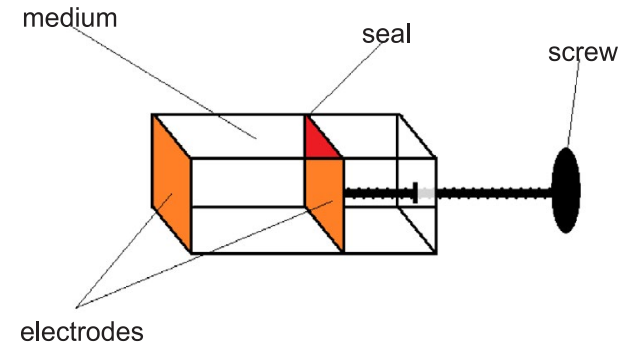

a)

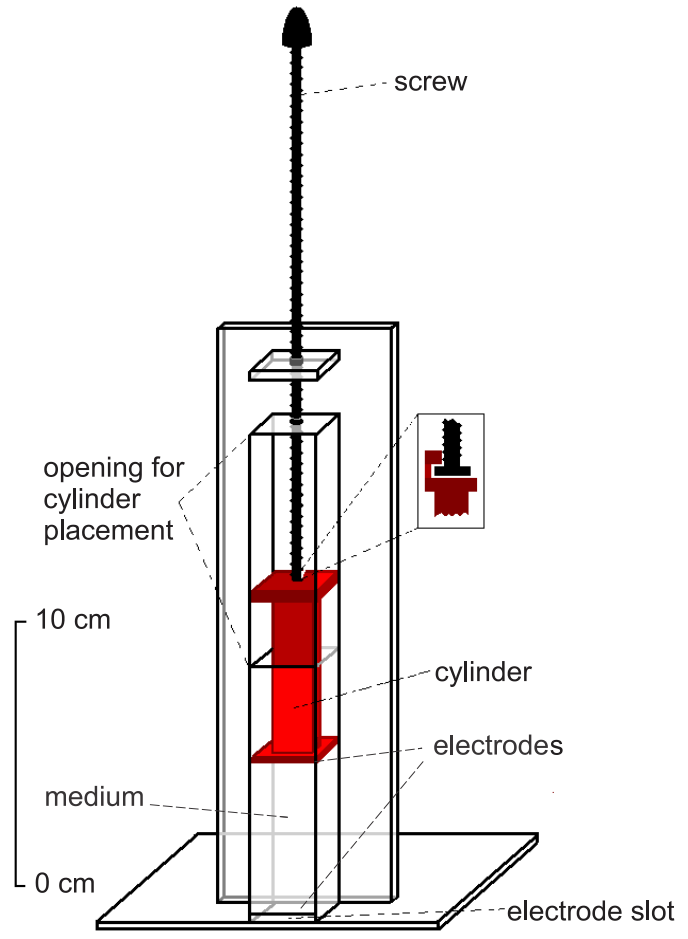

b)

Fig. 1: a) simplified view of the measurement cell, b) complete measurement setup.

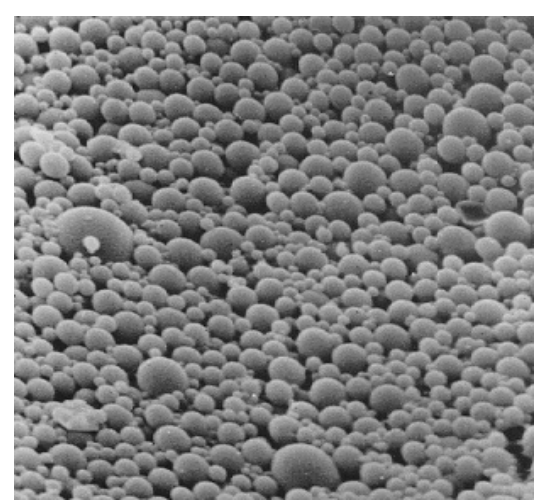

Fig. 2: A suspension of paraffin microcapsules used as a media.

A Quadtech 1920 precision LCR meter was used to measure the impedance. Though the LCR meter is capable of four electrode measurements, we used it in a classical two-electrode measurement configuration. The impedance was measured for 100 frequencies equally distributed in logarithmic scale in the frequency range from $10 \mathrm{~Hz}$ to 1 $\mathrm{MHz}$ A voltage measurement signal was set to $1 \mathrm{~V}$. One measurement cycle covering the 100 selected frequencies lasted about 1 minute.

\section{Separation of suspension and double layer impedances from the measured data}

In order to analyze the measurement results separately for the media and the double layer, the total impedance between the electrodes is described as a series connection of two double layer impedances, $\bar{Z}_{d l}$, (one for each suspension/electrode interface) and suspension impedance, $\bar{Z}_{s}$ (Figure 3):

$$
\bar{Z}=2 \bar{Z}_{d l}+\bar{Z}_{s}
$$

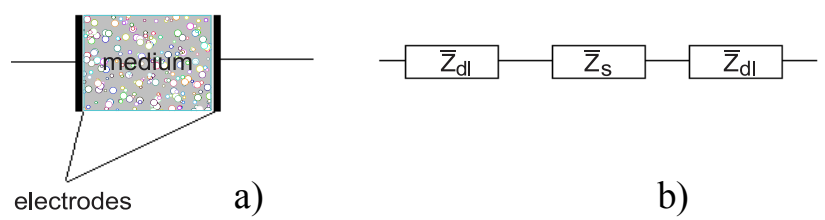

Fig. 3: a) medium (suspension) in the measurement cell and b) electrical equivalent of the measured impedance.

We assume that the measurement signal is small enough not to cause nonlinearities. We further assume that the double layer impedance does not depend on the distance between the electrodes. It thus remains unchanged with varying distance between the electrodes:

$$
\bar{Z}(\omega, l)=2 \bar{Z}_{d l}(\omega)+\bar{Z}_{s}(\omega, l),
$$

where $l$ is the distance between the electrodes. Furthermore, we assume that the impedance varies with distance between 
the electrodes as a linear function. This assumption holds if the media is linear and the electric field distribution in the media is homogeneous. In this case the media (suspension) impedance varies with distance between the electrodes as

$$
\bar{Z}_{s}(\omega, l)=\bar{f}(l)=f_{1}(l)+\mathrm{j} f_{2}(l)
$$

while the impedance of a double layer does not depend on the distance

$2 \bar{Z}_{d l}=\bar{n}=n_{1}+j n_{2}$.
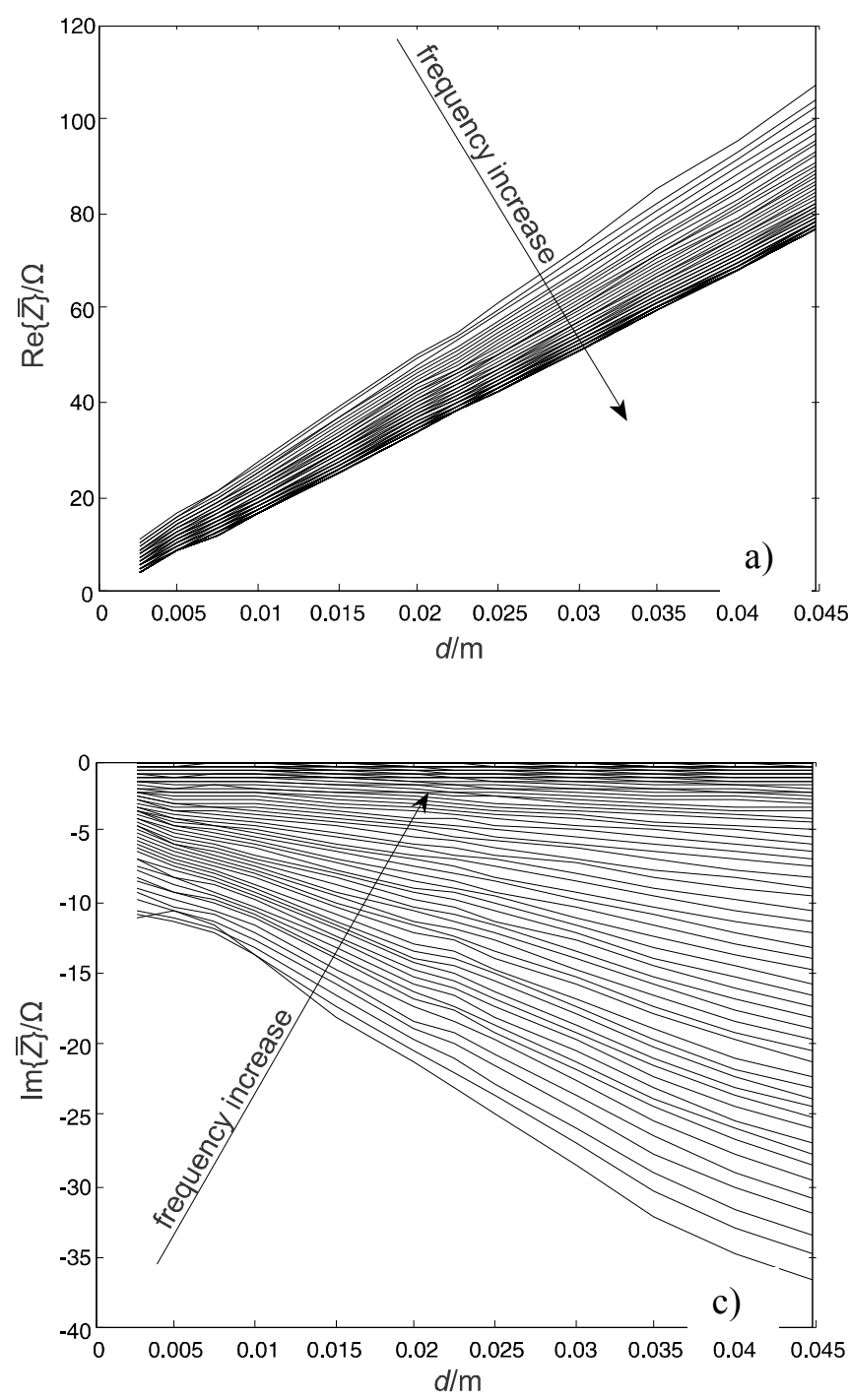

imaginary part and each is modeled as a linear function of the distance between the electrodes:

$$
\begin{aligned}
& \operatorname{Re}\{\bar{Z}(\omega, l)\}=n_{1}(\omega)+k_{1}(\omega) l \\
& \operatorname{Im}\{\bar{Z}(\omega, l)\}=n_{2}(\omega)+k_{2}(\omega) l
\end{aligned}
$$

The values $n_{1}, n_{2}, k_{1}, k_{2}$ are extracted from measured data by curve fitting of the real and imaginary part of measured impedances at various distances between the electrodes (see Figure 4), using the least square method in MATLAB.
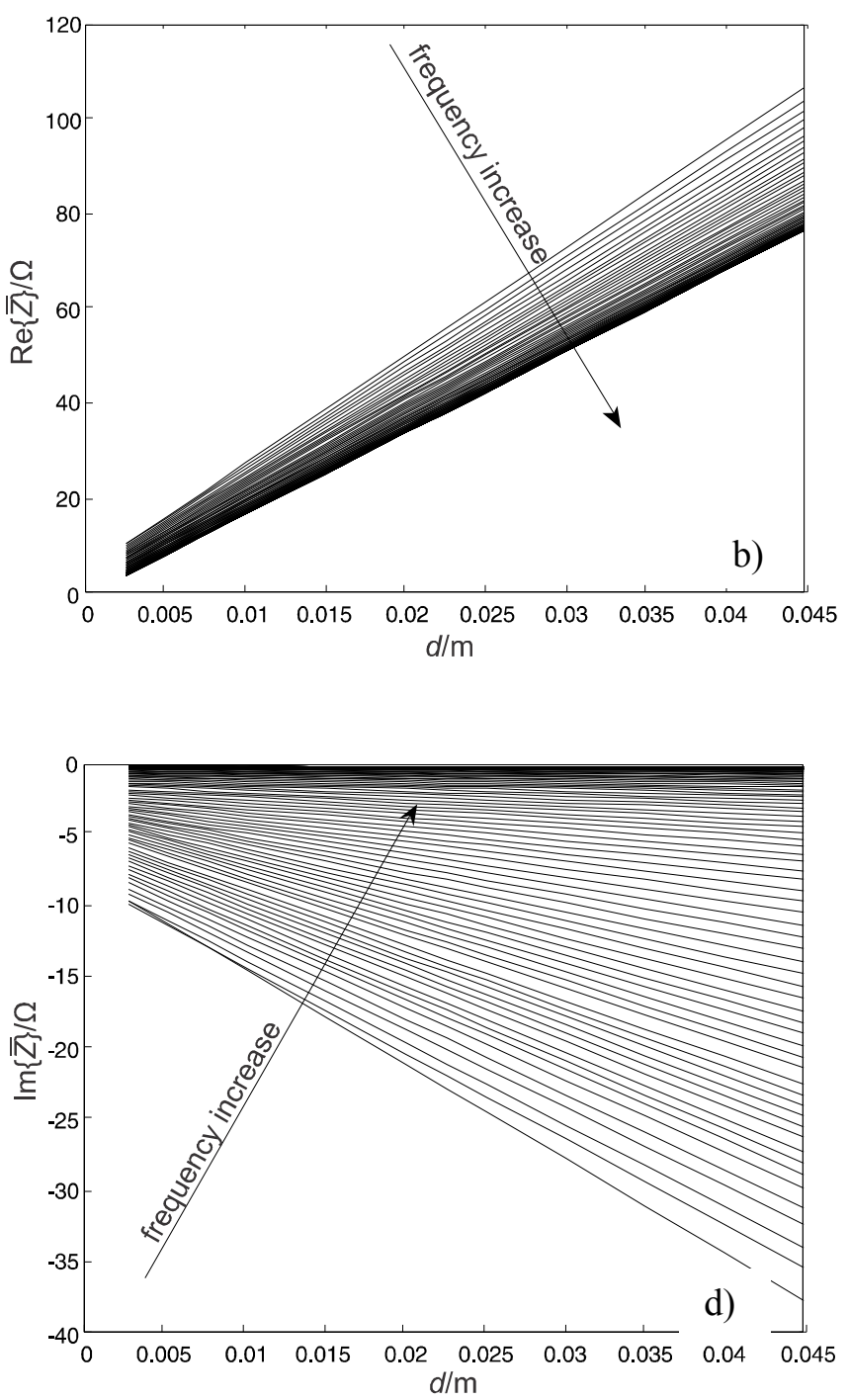

Fig. 4: Measured and fitted real (a and b) and imaginary ( $c$ and d) part as a function of the distance between the electrodes. For each measured frequency one fitted curve as a linear function of the distance between the electrodes is obtained.

The total impedance is thus

$$
\bar{Z}(\omega, l)=\left(f_{1}(l)+n_{1}\right)+\mathrm{j}\left(n_{1}+n_{2}\right)
$$

Figure 4 present measured and fitted real and imaginary part of the impedance between the electrodes for various frequencies and varying distances between the electrodes. The measured impedance is separated into the real and The impedances of the double layer and the suspension are thus obtained as

$$
\begin{gathered}
2 \bar{Z}_{d l}=\bar{n}=n_{1}+j n_{2} \\
\bar{Z}_{s}(\omega, l)=k_{1}(\omega) l+\mathrm{j} k_{2}(\omega) l
\end{gathered}
$$


Next the complex specific resistivity is defined as

$$
\bar{\rho}=\bar{Z} \frac{A}{l},
$$

where $A$ is the electrode area. Accordingly, the specific complex resistivity of the suspension is

$$
\bar{\rho}_{s}(\omega)=\left(k_{1}(\omega)+\mathrm{j} k_{2}(\omega)\right) A
$$

\section{Electrical models of a suspension and the double layer}

Once the impedances of the double layer and the media were obtained, an equivalent electrical circuit model was sought to explain the obtained (measured and extracted) results as best as possible.

Modeling can be done in two manners: the first is to develop a model to fit the measured data as closely as possible regardless of the characteristics of the material under investigation while the second is to find an electrical equivalent model that incorporates some material properties. Our approach towards a suitable model started from observations of the measured impedances in the complex plane. A typical plot of the extracted impedance of the paraffin suspension is shown in Figure 5. When the shape of the impedance in the complex plane is semicircular the impedance can be modeled as a parallel connection of a resistor and a capacitor. If a semicircle is obtained from admittance data, a more appropriate model is represented by a series connection of a resistor and a capacitor. With some additional elements more complex shapes can be obtained. Impedances of some basic connections are presented in Figure 6.

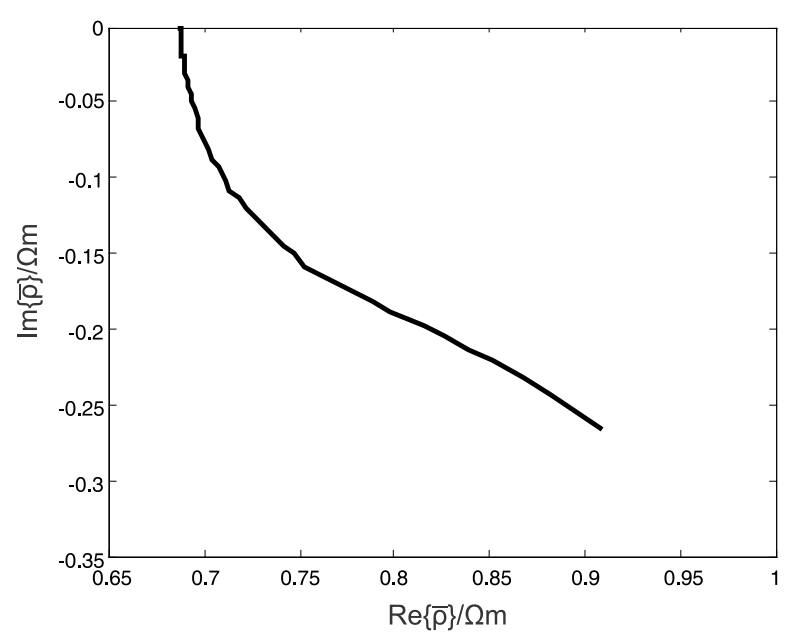

Fig. 5: Measured and extracted suspension impedance (specific complex resistivity) shown in the complex plane.

Clearly, in practice the impedance or admittance shapes can quite differ from simple semicircles shown in Figure 6. Neither the series nor the parallel connection of a resistor is usually sufficient to model the extracted impedance [17]. We thus started from a simple parallel connection of a resistor and a capacitor and developed further the model by adding additional elements and observe a reduction of the fitting error during the fitting procedure.

Some improvement of the model (through fitting error reduction) has been obtained by replacing the parallel capacitor with a constant phase element (CPE) element as shown in Figure 7. The final model that resulted in the smallest fitting error is presented in Figure 7. Some additional elements could further reduce the error but also increase the complexity of the model.

The analysis of the weight (importance) of the elements in the model revealed that in particular the elements $\mathrm{C}$ and $\mathrm{D}$ could represent specific resistivity and permittivity of the measured suspension. Element A influences the fit at low frequencies while elements $\mathrm{C}$ and $\mathrm{D}$ influence behavior of the model particularly at high frequencies.

Figure 8 presents a physics based model that is based on characteristics of a suspension of microcapsules. The paraffin microcapsules can be modeled as capacitors and resistors in parallel separated by resistors describing the media between the capsules. An additional resistor and capacitor pair in parallel describe the current flow in the liquid surrounding the capsules. This model is very similar to the model in Figure 7 obtained by starting from a simple parallel connection of a resistor and a capacitor. A comparison of the measured impedance and the impedance predicted from the model from Figure 8 is shown in Figure 9.

By fitting the measured data, the electrical parameters of the elements can be obtained. Table 1 presents fitted electrical parameters for elements A to E from Figure 7 for three different electrode materials $(\mathrm{Cu}, \mathrm{Ag}$ and $\mathrm{Au})$. Some differences in model parameters are expected to occur as a result of the fitting procedure in which no attempt was made to fix some values in advance.

\section{Modeling the double layer}

From measured data we separated the impedance due to the suspension and the double layer. Figure 10 presents extracted double layer admittance in a complex plane for various electrodes, in the cases where the media was only a $\mathrm{KCl}$ solution or a suspension with paraffin microcapsules. Clearly, a simple electrical model of the double layer could be a resistor in series with a CPE element. Inclusion of a CPE element instead of a capacitor is quite important since it is much more capable of fitting the flattened semicircle of admittance. 

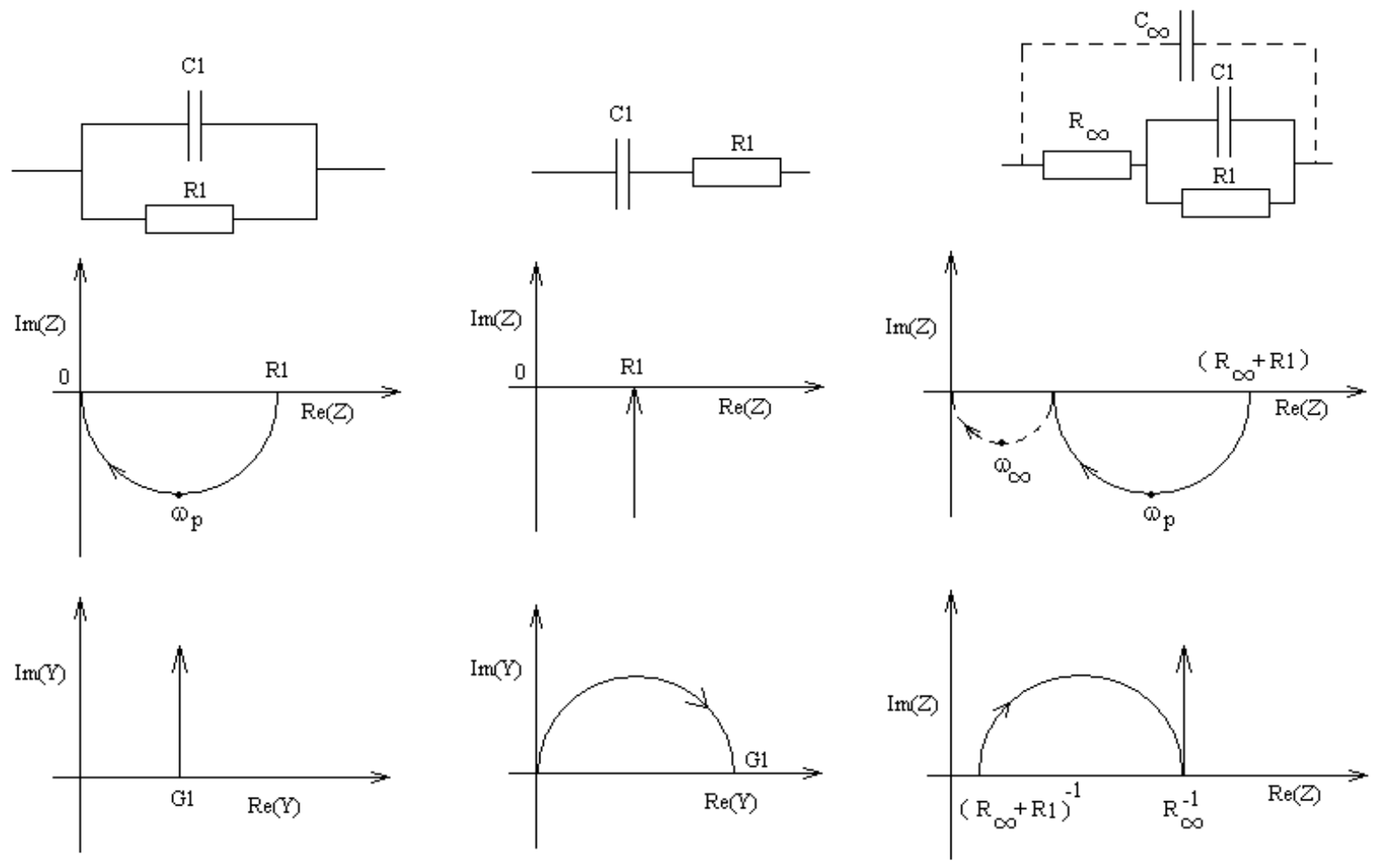

Fig. 6: Typical models with impedances and admittances in the complex plane. Semicircles of impedance denote parallel connection of a capacitor and resistor while semicircle of the admittance denotes series connection.

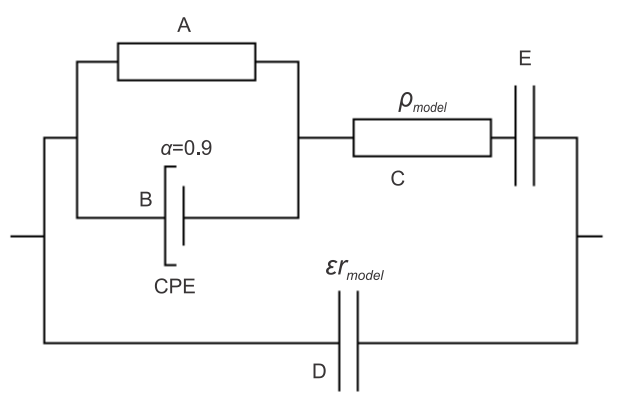

Table 1: Parameters extracted by fitting the model from Figure 7 to the measured and extracted impedance of a suspension.

\begin{tabular}{cccccc}
\hline Electrode material & $\begin{array}{c}\rho(\mathbf{A}) \\
{[\Omega \mathrm{m}]}\end{array}$ & $\begin{array}{c}\boldsymbol{\varepsilon}_{\mathrm{r}}(\mathbf{B}) \\
{ }_{10^{8}}\end{array}$ & $\begin{array}{c}\rho(\mathbf{C}) \\
{[\Omega \mathrm{m}]}\end{array}$ & $\begin{array}{c}\boldsymbol{\varepsilon}_{\mathrm{r}}(\mathbf{D}) \\
{ }^{*} \mathbf{1 0}^{9}\end{array}$ & $\boldsymbol{\varepsilon}_{\mathrm{r}}(\mathrm{E})$ \\
\hline $\mathrm{Cu}$ & 0,301 & 4,10 & 1,46626 & 3,94 & 59,91714 \\
$\mathrm{Ag}$ & 0,214 & 9,94 & 1,46438 & 2,59 & 51,46467 \\
$\mathrm{Au}$ & 0,279 & 11,19 & 1,46502 & 2,49 & 62,93998 \\
\hline
\end{tabular}

Fig. 7: The final model that modeled suspension impedance with smallest fitting error.
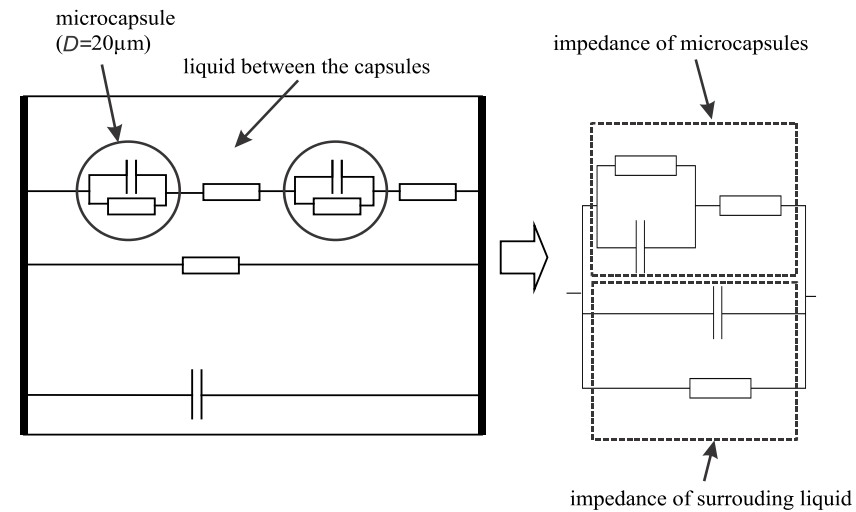

Fig. 8: Possible physics based model that can explain the electrical characteristics of a suspension of microcapsules.

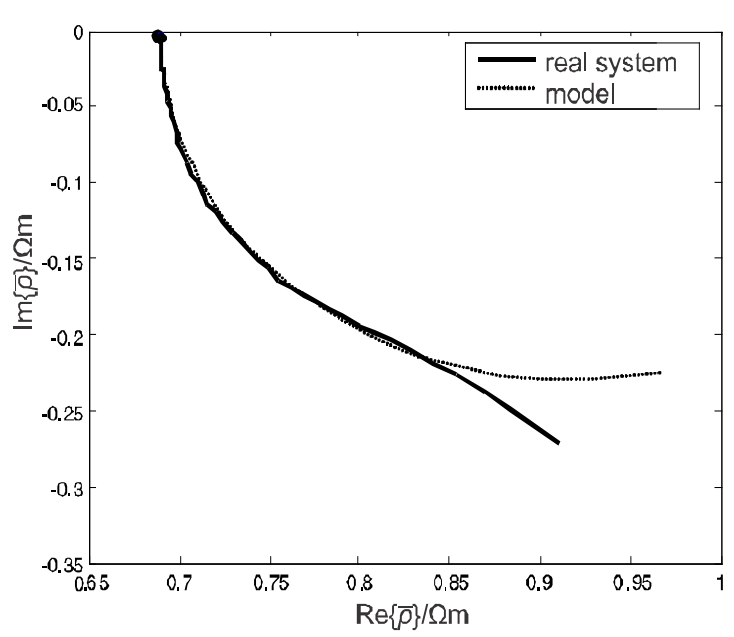

Fig. 9: The fitting between the physically based model and the measured impedance (specific complex resistivity) of a suspension. 


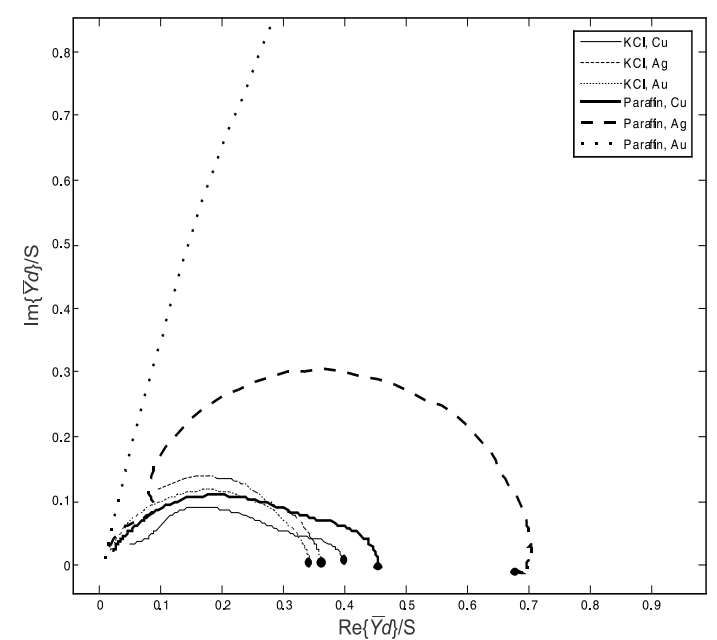

Fig. 10: Double layer impedance extracted from the measured total impedance for different electrodes and media with a suspension of microcapsules or only $\mathrm{KCl}$ solution.

Table 2 presents extracted parameters of a series resistor and CPE elements described by impedance $\bar{Y}_{C P E}=\mathrm{Q}^{\circ}(j \omega)^{n}$. The table reveals slightly different values of the parameters for different electrodes and also for cases in which a tested solution was only $0.1 \mathrm{M} \mathrm{KCl}$ or a suspension of microcapsules.

Table 2: Parameters determined by fitting the determined impedance of the double layer to the model of a CPE element in series with a resistor.

\begin{tabular}{cccc}
\hline Media, electrodes & $\alpha[\mathrm{CPE}] \mathrm{Rd}[\Omega]$ & $\mathbf{Q}^{\circ}[\mu \mathrm{F}]$ \\
\hline 0,1M KCl, $\mathrm{Cu}$ & 0,40 & 2,311 & 7,47 \\
0,1M KCl, Ag & 0,73 & 2,745 & 906,51 \\
0,1M KCl, Au & 0,70 & 2,905 & 37,37 \\
paraffin microcapsules, $\mathrm{Cu}$ & 0,53 & 2,087 & 9,00 \\
paraffin microcapsules, $\mathrm{Ag}$ & 0,68 & 1,510 & 72,78 \\
paraffin microcapsules, $\mathrm{Au}$ & 0,71 & 0,010 & 18,13 \\
\hline
\end{tabular}

\section{Parameter determination errors without inclusion of a double layer}

Is it important to take into account the impedance of the double layer? In a four-electrode measurement process we really do not have access to the impedance of the double layer. Its influence is avoided by placing the voltage measuring electrodes away from the electrodes imposing the current to the system. Our measurements enabled us to study both the impedance of a suspension and the double layer impedance. As a consequence we were able to compare the magnitudes of both at particular frequencies and infer the significance of each. Figure 11 presents the measured total impedance separated into the impedance of the double layer and the suspension impedance (top graphs). Each column presents results for one particular distance between the electrodes $(0.5 \mathrm{~cm}, 5 \mathrm{~cm}$ and $10 \mathrm{~cm})$.
The top row of plots presents the measured total impedance and extracted impedances of a suspension and double layer impedance. The middle row presents determined permittivity with or without inclusion of the double layer while the bottom row presents same but for the parameter specific resistivity. The results clearly indicate that double layer impedance is particularly important at lower frequencies and less at higher frequencies. This is due to particularly large capacitance of the double layer due to its thinness. These results are in line with many results from the vast literature on the double layer phenomena.

The importance of inclusion of the double layer in the model can be well represented by defining the relative error of parameter determination as

$$
\text { error }=\frac{x_{\text {meas }}-x_{\text {susp }}}{x_{\text {susp }}}
$$

where $\mathrm{x}$ can be any parameter determined without inclusion of the double layer ( $\left.x_{\text {meas }}\right)$ and by taking into account the double layer $x_{\text {susp }}$. Figures 12 and 13 present measurement errors dependent on frequency and the distance between the electrodes.

From the results we can conclude that the magnitude of the error depends on both parameters, the frequency of the measured signal as well as on the distance between the electrodes. Larger distance between the electrodes results in smaller determination error since the magnitude of the suspension impedance increases relatively to the impedance of the double layer. The measurement error is large at low frequencies particularly for the specific resistivity. For permittivity, larger frequencies result in larger errors

\section{Discussion on the developed lumped model}

We approached the modeling problem from two perspectives. One was to start from simplified models containing resistors and capacitors as presented in Figure 6 and increase its complexity until we were satisfied with the fit (reduction of the fitting error was not significant after incorporation of additional elements to the model). We then checked whether the fitting error can be reduced by replacing the capacitors with the CPE elements. With this approach we ended with a model presented in Figure 7. The obtained model does not account for DC conductivity but it was an optimal fit for the extracted data that were obtained for frequencies from $10 \mathrm{~Hz}$ to $1 \mathrm{MHz}$. These data do not cover DC conduction. Furthermore, one should note that relative permittivity of the capacitor E in Figure 7 is very large $\left(10^{9}\right)$ so it has large susceptance $(\omega C)$ at very low frequencies. On the other hand, the physics based approach yielded the model presented in Figure 8, which enabled DC conduction and can resemble some material properties of the media and different types of current flow. 

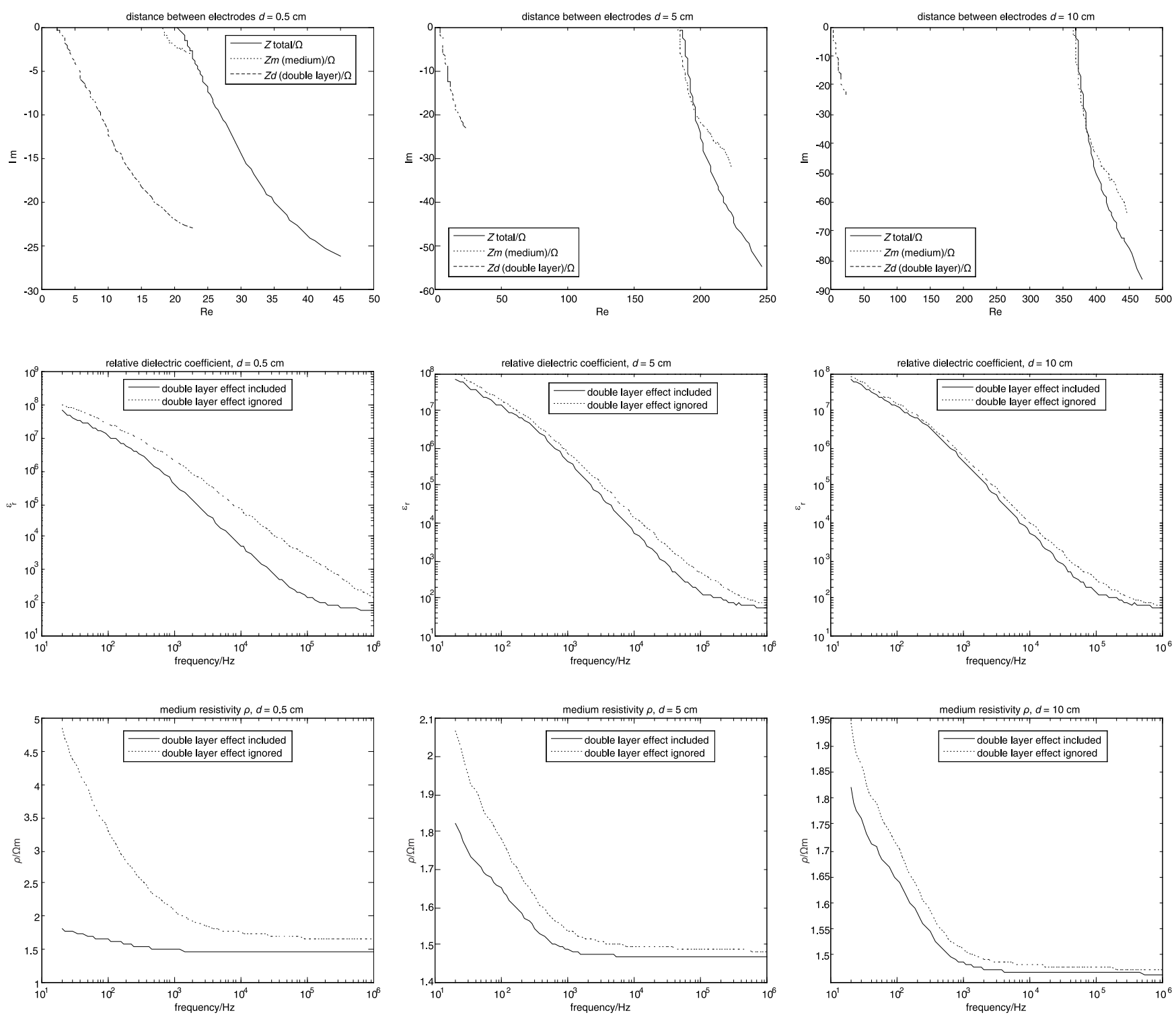

Fig. 11: Comparison of the impedance due to the suspension and the double layer. The columns from left to right present results for distance between the electrodes $0.5 \mathrm{~cm}, 5 \mathrm{~cm}$ and $10 \mathrm{~cm}$, respectively. Rows from top to bottom represent graphs of (total and separated to double layer and suspension) absolute values of impedance, permittivity and specific resistivity, respectively.

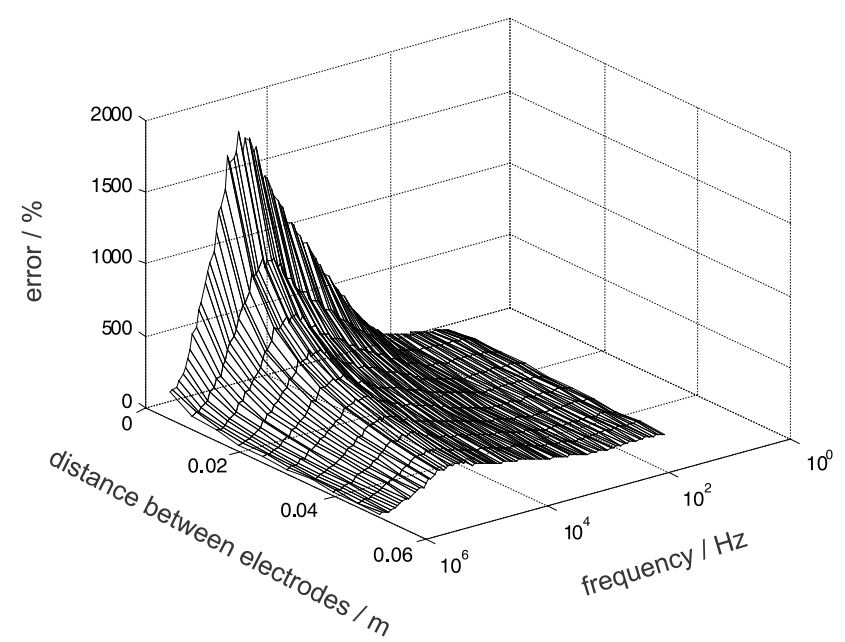

Fig. 12: Solution permittivity determination error in case the double layer is not taken into account. Frequency range is from $20 \mathrm{~Hz}$ to $1 \mathrm{MHz}$, the distance between the electrodes is from 5 to $45 \mathrm{~mm}$. 


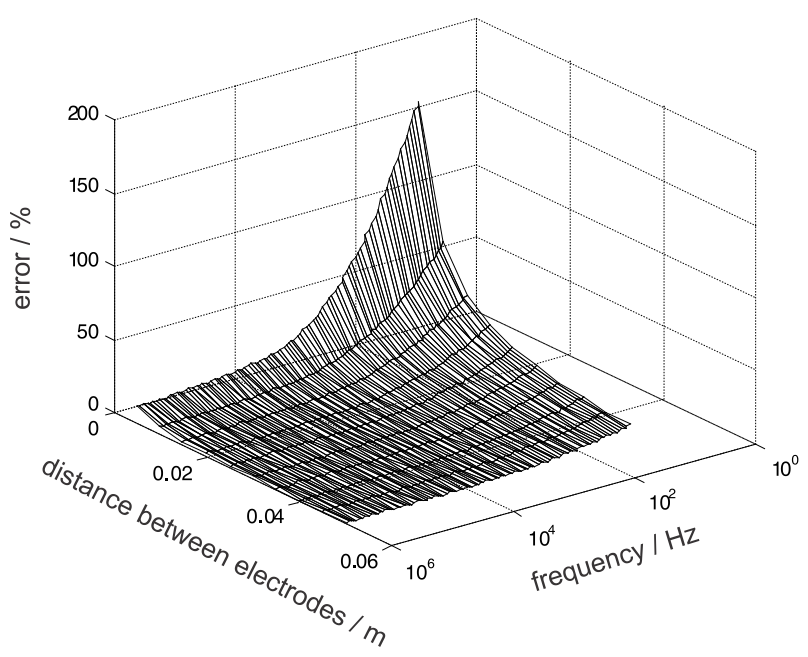

Fig. 13: Solution specific resistivity determination error in case the double layer is not taken into account. Frequency range is from $20 \mathrm{~Hz}$ to $1 \mathrm{MHz}$, the distance between the electrodes is from 5 to $45 \mathrm{~mm}$.

Both developed models are an approximation for description of current conduction through a suspension of microcapsules. Inclusion of some additional elements could thus reduce the fitting error but on the expense of increased complexity of the model (inclusion of additional lumped elements).

\section{Conclusion}

We have demonstrated a possible technique for extraction of a suspension and double layer impedance by developing a measurement cell that is able to vary the distance between the electrodes. A suspension of paraffin microcapsules was used as a measurement sample media. It was assumed that the double layer impedance is not affected by the variation of the distance between the electrodes while the suspension impedance increased linearly with the distance. Fitting the measured data to the linear curve the impedances that correspond to the suspension and those from the double layer could be separated and accounted for. The suspension impedance was further modeled by a suitable circuit equivalent model starting from a simple parallel RC model and advancing to a more complex model that resulted in the smallest fitting error possible, enabling the determination of important suspension parameters such as permittivity and specific resistivity. The error in determining these parameters has been studied by comparing them to the case in which a double layer was not taken into account. Significant errors are obtained for specific resistivity for small distances between the electrodes and low frequencies. The permittivity determination error is more pronounced at frequencies around $10 \mathrm{kHz}$ and small distances between the electrodes.

\section{References}

1. Grimnes S, Martinsen OG, Bioimpedance and Bioelectricity Basics, Academic Press, 2000.

2. Geddes LA. Historical evolution of circuit models for the electrode-electrolyte interface, Annals of Biomedical Engineering 25(1), pp. 1-14, 1997. http://dx.doi.org/10.1007/BF02738534

3. Fricke H, Curtis HJ, The electrical impedance of hemolized suspensions of mamalian erythrocytes, J. Gen. Physol 18, 821-836, 1934-1935. http://dx.doi.org/10.1085/jgp.18.6.821

4. Geddes LA, Measurement of electrolytic resistivity and electrode-electrolyte impedance using variable length conductivity cell, Instr. Sci Tech 4, 157-168, 1972. http://dx.doi.org/10.1080/10739147208543345

5. Jarvis JB et al. Measuring permittivity and permeability of lossy materials, NIST Technical Note 1536, NIST, Boulder, CO, USA, 2004.

6. Schwan HP, Linear and nonlinear electrode polarization and biological materials, Ann. Biomed. Eng., vol. 20, pp. 269-88, 1992. http://dx.doi.org/10.1007/BF02368531

7. Grimnes S, Martinsen ØG, Sources of error in tetrapolar impedance measurements on biomaterials and other ionic conductors, J. Phys. D. App. Phys., vol. 40, pp. 9-14, 2007. http://dx.doi.org/10.1088/0022-3727/40/1/S02

8. Schwan HP, Ferris CD, Four electrode null techniques, Rev. Sci. Instrum., vol. 39, pp. 481-5, 1968. http://dx.doi.org/10.1063/1.1683413

9. Geddes LA, Da Costa CP, Wise G, The impedance of stainless steel electrodes, Med. Biol. Eng., vol. 9, pp. 511-21, 1971. http://dx.doi.org/10.1007/BF02474708

10. Padmaraj D, Miller JH, Wosik J, Zagozdzon-Wosik W, Reduction of electrode polarization capacitance in lowfrequency impedance spectroscopy by using mesh electrodes, Biosensors \& bioelectronics, 29(1), 13-7, 2011. http://dx.doi.org/10.1016/j.bios.2011.06.050

11. Kalvøy H, Johnsen GK, Martinsen OG, Grimnes S, New method for separation of electrode polarization impedance from measured tissue impedance, The Open Biomedical Engineering Journal 5, pp. 8-13, 2011.

12. Hill NE, Vaughan VE, Price AH, Davies M, Dielectric properties and molecular behavior, Van Nostrand Reinchold Company, GB, 1969.

13. Raju GG, Dielectrics in electric field, Marcel Dekker, 2003. http://dx.doi.org/10.1201/9780203912270

14. Barsoukov E, Macdonald JR, Impedance spectroscopy theory, experiment and applications, Wiley-Interscience, USA, 2005. http://dx.doi.org/10.1002/0471716243

15. Cirkel P A., van der Ploeg JPM, Koper GJM, Electrode effects in dielectric spectroscopy of colloidal suspensions, Physica A: Statistical Mechanics and its Applications, vol 235(1-2), pp. 269-78, 1997. 
16. Fricke H, The theory of electrolytic polarization, Phil. Mag. 14, pp. 310-318, 1932.

17. Yardley JE, Todd R, Nicholson DJ, Barrett J, Kell DB, Davey CL, Correction of the influence of baseline artefacts and electrode polarisation on dielectric spectra, Bioelectrochemistry 1(1), pp. 53-65, 2000. http://dx.doi.org/10.1016/S0302-4598(99)00069-0

18. Bordi F, Cametti C, Gili T, Reduction of the contribution of electrode polarization effects in the radiowave dielectric measurements of highly conductive biological cell suspensions, Bioelectrochemistry, vol 54(1), pp. 53-61, 2001. http://dx.doi.org/10.1016/S1567-5394(01)00110-4

19. Mirtaheri P, Grimnes S, Martinsen OG, Electrode polarization impedance in weak $\mathrm{NaCl}$ aqueous solutions, IEEE Transactions on Biomedical Engineering, vol 52(12), pp. 2093-9, 2005.

http://dx.doi.org/10.1109/TBME.2005.857639

20. Benita Simon, Microencapsulation: Methods and Industrial Applications, Marcel Dekker, Inc., New York. 1996. 